\title{
THREE-VARIABLE ALTERNATING TRIGONOMETRIC FUNCTIONS AND CORRESPONDING FOURIER TRANSFORMS
}

\author{
Agata BezubiK ${ }^{a}$, Severin Pošta ${ }^{b, *}$ \\ ${ }^{a}$ Institute of Mathematics, University of Biatystok, Akademicka 2, 15-267 Biatystok, Poland \\ ${ }^{b}$ Department of mathematics, Faculty of nuclear sciences and physical engineering, Czech Technical University \\ in Prague, Trojanova 13, 12000 Prague, Czech Republic \\ * corresponding author: severin.posta@fjfi.cvut.cz
}

\begin{abstract}
The common trigonometric functions admit generalizations to any higher dimension. In this paper, we restrict ourselves to three dimensional generalization only, focusing on alternating case in detail. Many specific properties of this new class of special functions are studied, such as the orthogonalities, both the continuous one and the discrete one on the $3 D$ lattice of any density, discrete and continuous Fourier transforms, and others. Rapidly increasing precision of the interpolation with increasing density of the $3 D$ lattice is shown in an example.
\end{abstract}

KEYWORDS: trigonometric functions; orthogonal polynomials.

\section{INTRODUCTION}

There are many applications of functions which are symmetric or antisymmetric with respect to the symmetry group $S_{n}$ in mathematics. They appear for example in quantum theory or in theory of integrable systems. In [1] (anti)symmetric multivariate exponential functions were defined and corresponding Fourier transforms were given. These functions were examined in detail in dimension two and three in [2]. The natural question which arises is the restriction of the symmetry to the subgroup $A_{n}$ of $S_{n}$ consisting of transformations $w$ with $\operatorname{det} w=1$. Such functions were examined in [3] for $A_{n}$ involving the general number $n$, and in [4] in greater detail concerning the smallest possible $n$, namely $n=3$.

The exponential functions are not the only functions which admit symmetric, antisymmetric and alternating generalizations to higher dimensions. Their odd and even counterparts, sines and cosines, admit similar generalizations and fulfil many analogical properties as pure exponentials. Symmetric and antisymmetric case in general was covered in [5], alternating case was covered in [6]. Two-dimensional symmetric and antisymmetric generalizations were studied in detail in [7, 8].

The aim of this paper is to study in detail the alternating generalization of sine and cosine functions in dimension three.

(Anti)symmetric multivariate sine and cosine functions, considered in [5, 7, 8], as well as alternating multivariate sine and cosine functions, studied in [6] and in this paper, are closely related to symmetric and antisymmetric orbit functions studied in [9-11]. Because the definition of the considered functions and orbit functions are similar (roughly speaking, the exponentials are replaced by sines and cosines), they satisfy the same or similar properties, namely symmetry relations, reductions to the dominant or semidominant forms, periodicity etc. The discrete Fourier transforms of sine and cosine functions can be derived with the help of relations valid for exponential functions. 8 . The restriction of the functions to three variables allows us to be more specific about the details of their properties in the following sections. This is most notable when describing their discretization and orthogonality relations.

The paper consists of several parts. After the first part, devoted to the definition of three dimensional alternating sine and cosine functions and their basic properties, come the parts describing their continuous orthogonality relations, their decomposition rules and the two parts treating four kinds of discrete cosine and sine transforms.

\section{Definition}

Three dimensional alternating sine functions $\sin _{(\lambda, \mu, \nu)}: \mathbb{R}^{3} \rightarrow \mathbb{R}$ have the following explicit form [6]:

$$
\begin{array}{r}
\sin _{(\lambda, \mu, \nu)}(x, y, z)=\frac{1}{2}\left|\begin{array}{lll}
\sin 2 \pi \lambda x & \sin 2 \pi \lambda y & \sin 2 \pi \lambda z \\
\sin 2 \pi \mu x & \sin 2 \pi \mu y & \sin 2 \pi \mu z \\
\sin 2 \pi \nu x & \sin 2 \pi \nu y & \sin 2 \pi \nu z
\end{array}\right|+\frac{1}{2}\left|\begin{array}{rrr}
\sin 2 \pi \lambda x & \sin 2 \pi \lambda y & \sin 2 \pi \lambda z \\
\sin 2 \pi \mu x & \sin 2 \pi \mu y & \sin 2 \pi \mu z \\
\sin 2 \pi \nu x & \sin 2 \pi \nu y & \sin 2 \pi \nu z
\end{array}\right| \\
=\sin 2 \pi \lambda x \sin 2 \pi \mu y \sin 2 \pi \nu z+\sin 2 \pi \lambda z \sin 2 \pi \mu x \sin 2 \pi \nu y+\sin 2 \pi \lambda y \sin 2 \pi \mu z \sin 2 \pi \nu x \\
x, y, z, \lambda, \mu, \nu \in \mathbb{R}
\end{array}
$$


where the second determinant with superscipt ${ }^{+}$stands for permanent [12], which is symmetric with respect to permutations of its rows and columns.

Three dimensional alternating cosine functions $\cos _{(\lambda, \mu, \nu)}: \mathbb{R}^{3} \rightarrow \mathbb{R}$ are defined similarly:

$$
\begin{array}{r}
\cos (\lambda, \mu, \nu) \\
(x, y, z)=\frac{1}{2}\left|\begin{array}{lll}
\cos 2 \pi \lambda x & \cos 2 \pi \lambda y & \cos 2 \pi \lambda z \\
\cos 2 \pi \mu x & \cos 2 \pi \mu y & \cos 2 \pi \mu z \\
\cos 2 \pi \nu x & \cos 2 \pi \nu y & \cos 2 \pi \nu z
\end{array}\right|+\frac{1}{2}\left|\begin{array}{lll}
\cos 2 \pi \lambda x & \cos 2 \pi \lambda y & \cos 2 \pi \lambda z \\
\cos 2 \pi \mu x & \cos 2 \pi \mu y & \cos 2 \pi \mu z \\
\cos 2 \pi \nu x & \cos 2 \pi \nu y & \cos 2 \pi \nu z
\end{array}\right|^{+} \\
=\cos 2 \pi \lambda x \cos 2 \pi \mu y \cos 2 \pi \nu z+\cos 2 \pi \lambda z \cos 2 \pi \mu x \cos 2 \pi \nu y+\cos 2 \pi \lambda y \cos 2 \pi \mu z \cos 2 \pi \nu x \\
x, y, z, \lambda, \mu, \nu \in \mathbb{R} .
\end{array}
$$

From (1) we immediately see the (rotational) symmetry of $\sin _{(\lambda, \mu, \nu)}(x, y, z)$ with respect to cyclic permutations of variables $(x, y, z)$ and $(\lambda, \mu, \nu)$ :

$$
\sin _{(\lambda, \mu, \nu)}(x, y, z)=\sin _{(\lambda, \mu, \nu)}(z, x, y)=\sin _{(\lambda, \mu, \nu)}(y, z, x)=\sin _{(\nu, \lambda, \mu)}(x, y, z)=\sin _{(\mu, \nu, \lambda)}(x, y, z) .
$$

From 2 we have similar symmetries for $\cos _{(\lambda, \mu, \nu)}(x, y, z)$ :

$$
\cos _{(\lambda, \mu, \nu)}(x, y, z)=\cos _{(\lambda, \mu, \nu)}(z, x, y)=\cos _{(\lambda, \mu, \nu)}(y, z, x)=\cos _{(\nu, \lambda, \mu)}(x, y, z)=\cos _{(\mu, \nu, \lambda)}(x, y, z) .
$$

The rotational symmetries above allow us to put any real triple $(\lambda, \mu, \nu)$ to the canonical form. For example, we may restrict ourselves to the functions $\sin _{(\lambda, \mu, \nu)}$ and $\cos _{(\lambda, \mu, \nu)}$ with so called semidominant $(\lambda, \mu, \nu)$, that is, triples $(\lambda, \mu, \nu)$ where $\lambda \geq \mu \geq \nu$ or $\mu>\lambda>\nu$ holds.

The functions $\sin _{(k, l, m)}$ and $\cos (k, l, m)$ with $k, l, m \in \mathbb{Z}$ have additional symmetries induced by the periodicity of sine and cosine functions:

$$
\begin{array}{ll}
\sin _{(k, l, m)}(x+r, y+s, z+t)=\sin _{(k, l, m)}(x, y, z), & r, s, t \in \mathbb{Z}, \\
\cos _{(k, l, m)}(x+r, y+s, z+t)=\cos _{(k, l, m)}(x, y, z), & r, s, t \in \mathbb{Z} .
\end{array}
$$

Another symmetries of $\sin _{(\lambda, \mu, \nu)}$ and $\cos _{(\lambda, \mu, \nu)}$ follow from the well known fact that ordinary sine and cosine functions of one variable are odd and even functions, respectively. Therefore for all $\lambda, \mu, \nu$ and $x, y, z$ we have

$$
\begin{gathered}
\sin _{(\lambda, \mu, \nu)}(-x, y, z)=\sin _{(\lambda, \mu, \nu)}(x,-y, z)=\sin _{(\lambda, \mu, \nu)}(x, y,-z)=-\sin _{(\lambda, \mu, \nu)}(x, y, z), \\
\cos _{(\lambda, \mu, \nu)}(-x, y, z)=\cos _{(\lambda, \mu, \nu)}(x,-y, z)=\cos _{(\lambda, \mu, \nu)}(x, y,-z)=\cos _{(\lambda, \mu, \nu)}(x, y, z) .
\end{gathered}
$$

There is also duality of $\sin _{(\lambda, \mu, \nu)}$ and $\cos _{(\lambda, \mu, \nu)}$ functions,

$$
\sin _{(\lambda, \mu, \nu)}(x, y, z)=\sin _{(x, y, z)}(\lambda, \mu, \nu), \cos _{(\lambda, \mu, \nu)}(x, y, z)=\cos _{(x, y, z)}(\lambda, \mu, \nu) .
$$

Moreover,

$$
\sin _{(k, l, m)}(x, y, z)=0 \quad \text { when } \quad k=0 \text { or } l=0 \text { or } m=0,
$$

therefore we can exclude $\sin _{(k, l, m)}$ with at least one index being zero from our considerations.

The relations (3)- 6 imply that it is sufficient to restrict to the following families of functions:

$$
\sin _{(k, l, m)}, \quad k, l, m \in \mathbb{N}, k \geq l \geq m \text { or } l>k>m,
$$

and

$$
\cos _{(k, l, m)}, \quad k, l, m \in \mathbb{Z}, k \geq l \geq m \geq 0 \text { or } l>k>m \geq 0,
$$

and to consider them on the closure of the fundamental domain $F\left(\tilde{A}_{3}^{\text {aff }}\right)[\underline{6}$ only. This fundamental domain of the dual affine group of $A_{3}$ can be chosen in $3 \mathrm{D}$ to be equal to the part of the cube shown in Fig. 1

$$
F\left(\tilde{A}_{3}^{\mathrm{aff}}\right)=\left\{(x, y, z) \in\left(0, \frac{1}{2}\right) \times\left(0, \frac{1}{2}\right) \times\left(0, \frac{1}{2}\right) \mid x>z, y>z\right\} .
$$

\section{Continuous orthogonality}

The alternating trigonometric functions $\sin _{(k, l, m)}$ resp. $\cos _{(k, l, m)}$ are pairwise orthogonal on $F\left(\tilde{A}_{3}^{\text {aff }}\right)$. Let us denote

$$
G_{k l m}= \begin{cases}3 & \text { if } k=l=m \\ 1 & \text { otherwise }\end{cases}
$$




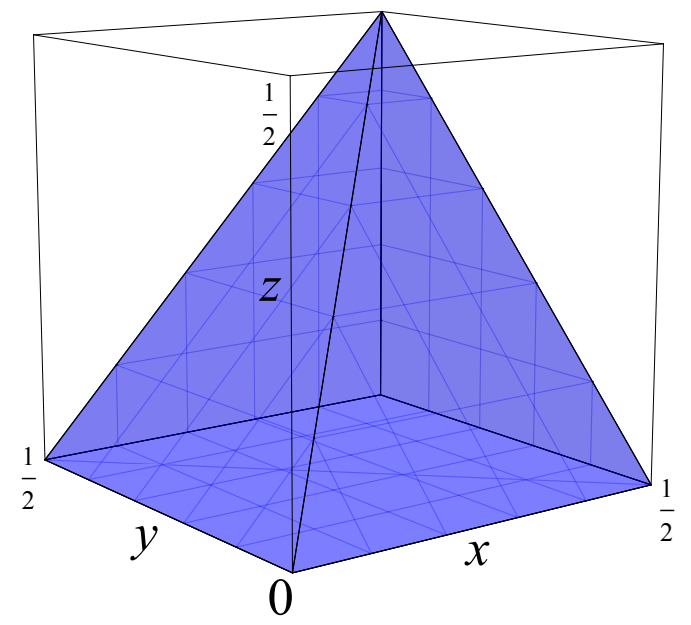

Figure 1. Fundamental domain $F\left(\tilde{A}_{3}^{\text {aff }}\right)$

Then we have

$$
\int_{F\left(\tilde{A}_{3}^{\text {aff }}\right)} \sin _{(k, l, m)}(x, y, z) \sin _{\left(k^{\prime}, l^{\prime}, m^{\prime}\right)}(x, y, z) \mathrm{d} x \mathrm{~d} y \mathrm{~d} z=\frac{G_{k l m}}{64} \delta_{k k^{\prime}} \delta_{l l^{\prime}} \delta_{m m^{\prime}},
$$

where $k, l, m, k^{\prime}, l^{\prime}, m^{\prime} \in \mathbb{N}, k \geq l \geq m$ or $l>k>m, k^{\prime} \geq l^{\prime} \geq m^{\prime}$ or $l^{\prime}>k^{\prime}>m^{\prime}$, and

$$
\int_{F\left(\tilde{A}_{3}^{\text {aff }}\right)} \cos _{(k, l, m)}(x, y, z) \cos _{\left(k^{\prime}, l^{\prime}, m^{\prime}\right)}(x, y, z) \mathrm{d} x \mathrm{~d} y \mathrm{~d} z=\frac{G_{k l m}}{64} \delta_{k k^{\prime}} \delta_{l l^{\prime}} \delta_{m m^{\prime}},
$$

where $k, l, m, k^{\prime}, l^{\prime}, m^{\prime} \in\{0,1,2, \ldots\}, k \geq l \geq m$ or $l>k>m, k^{\prime} \geq l^{\prime} \geq m^{\prime}$ or $l^{\prime}>k^{\prime}>m^{\prime}$.

Let us have a function $f: \mathbb{R}^{3} \rightarrow \mathbb{R}$ with the following properties: rotational symmetry, that is,

$$
f(x, y, z)=f(y, z, x)=f(z, x, y) ;
$$

periodic, that is

$$
f(x+r, y+s, z+t)=f(x, y, z) \text { for all } r, s, t \in \mathbb{Z}
$$

and odd in each variable, that is,

$$
f(-x, y, z)=f(x,-y, z)=f(x, y,-z)=-f(x, y, z) .
$$

Let $f$ be sufficiently smooth. Then it can be expanded in terms of the alternating sine functions, respectively, using formulas

$$
\begin{gathered}
f(x, y, z)=\sum_{\substack{k, l, m \geq 1 \\
k \geq l \geq m \text { or } l>k>m}} c_{k l m} \sin _{(k, l, m)}(x, y, z), \\
c_{k l m}=64 G_{k l m}^{-1} \int_{F\left(\tilde{A}_{3}^{\text {aff }}\right)} f(x, y, z) \sin _{(k, l, m)}(x, y, z) \mathrm{d} x \mathrm{~d} y \mathrm{~d} z .
\end{gathered}
$$

Similarly, any function $\tilde{f}: \mathbb{R}^{3} \rightarrow \mathbb{R}$ with the symmetries

$$
\begin{gathered}
\tilde{f}(x, y, z)=f(y, z, x)=f(z, x, y), \\
\tilde{f}(x+r, y+s, z+t)=f(x, y, z) \text { for all } r, s, t \in \mathbb{Z}, \\
\tilde{f}(-x, y, z)=f(x,-y, z)=f(x, y,-z)=f(x, y, z),
\end{gathered}
$$

and sufficiently smooth can be expanded in terms of the alternating cosine functions. The expansion is done using the formulas

$$
\begin{gathered}
\tilde{f}(x, y, z)=\sum_{\substack{k, l, m \geq 0 \\
k \geq l \geq m \text { or } l>k>m}} \tilde{c}_{k l m} \cos _{(k, l, m)}(x, y, z), \\
\tilde{c}_{k l m}=64 G_{k l m}^{-1} \int_{F\left(\tilde{A}_{3}^{\text {aff }}\right)} \tilde{f}(x, y, z) \cos _{(k, l, m)}(x, y, z) \mathrm{d} x \mathrm{~d} y \mathrm{~d} z .
\end{gathered}
$$




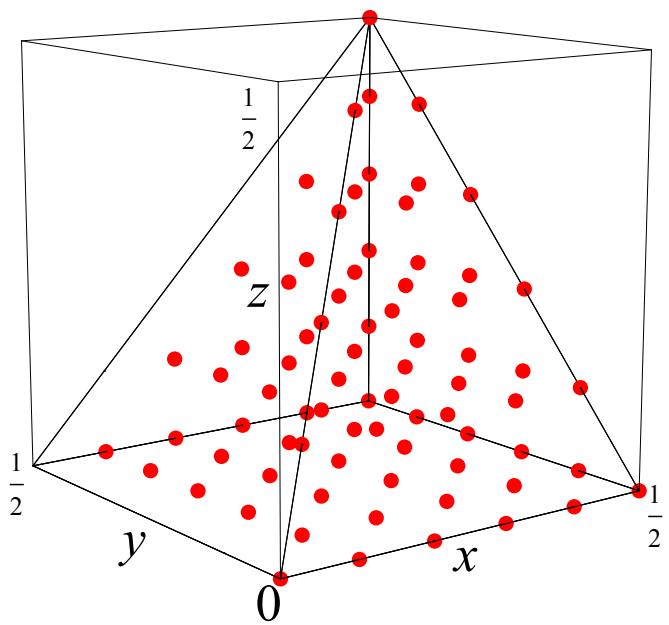

FiguRE 2. Finite grid of points in $F\left(\tilde{A}_{3}^{\text {aff }}\right)$

\section{Product DeComposition}

The product of two alternating cosines, $\cos _{(\lambda, \mu, \nu)} \cos _{\left(\lambda^{\prime}, \mu^{\prime}, \nu^{\prime}\right)}$ (we omit general argument $(x, y, z)$ here) can be decomposed into the sum of alternating cosines using the following formulas. The decomposition can serve e. g. for the derivation of recursion relations for the considered functions.

We have

$$
\begin{array}{r}
\cos _{(\lambda, \mu, \nu)} \cos \left(\lambda^{\prime}, \mu^{\prime}, \nu^{\prime}\right)=\frac{1}{8}\left(\cos _{\left(\lambda-\lambda^{\prime}, \mu-\mu^{\prime}, \nu-\nu^{\prime}\right)}+\cos _{\left(\lambda-\lambda^{\prime}, \mu-\mu^{\prime}, \nu+\nu^{\prime}\right)}+\cos _{\left(\lambda-\lambda^{\prime}, \mu+\mu^{\prime}, \nu-\nu^{\prime}\right)}\right. \\
+\cos _{\left(\lambda-\lambda^{\prime}, \mu+\mu^{\prime}, \nu+\nu^{\prime}\right)}+\cos \left(\lambda+\lambda^{\prime}, \mu-\mu^{\prime}, \nu-\nu^{\prime}\right)+\cos \left(\lambda+\lambda^{\prime}, \mu-\mu^{\prime}, \nu+\nu^{\prime}\right)+\cos \left(\lambda+\lambda^{\prime}, \mu+\mu^{\prime}, \nu-\nu^{\prime}\right)+\cos \left(\lambda+\lambda^{\prime}, \mu+\mu^{\prime}, \nu+\nu^{\prime}\right) \\
+\cos \left(\lambda-\mu^{\prime}, \mu-\nu^{\prime}, \nu-\lambda^{\prime}\right)+\cos \left(\lambda-\mu^{\prime}, \mu-\nu^{\prime}, \lambda^{\prime}+\nu\right)+\cos \left(\lambda-\mu^{\prime}, \mu+\nu^{\prime}, \nu-\lambda^{\prime}\right)+\cos \left(\lambda-\mu^{\prime}, \mu+\nu^{\prime}, \lambda^{\prime}+\nu\right)+\cos \left(\lambda+\mu^{\prime}, \mu-\nu^{\prime}, \nu-\lambda^{\prime}\right) \\
+\cos _{\left(\lambda+\mu^{\prime}, \mu-\nu^{\prime}, \lambda^{\prime}+\nu\right)}+\cos \left(\lambda+\mu^{\prime}, \mu+\nu^{\prime}, \nu-\lambda^{\prime}\right)+\cos \left(\lambda+\mu^{\prime}, \mu+\nu^{\prime}, \lambda^{\prime}+\nu\right)+\cos \left(\lambda-\nu^{\prime}, \mu-\lambda^{\prime}, \nu-\mu^{\prime}\right)+\cos \left(\lambda-\nu^{\prime}, \mu-\lambda^{\prime}, \mu^{\prime}+\nu\right) \\
+\cos \left(\lambda-\nu^{\prime}, \lambda^{\prime}+\mu, \nu-\mu^{\prime}\right)+\cos \left(\lambda-\nu^{\prime}, \lambda^{\prime}+\mu, \mu^{\prime}+\nu\right)+\cos \left(\lambda+\nu^{\prime}, \mu-\lambda^{\prime}, \nu-\mu^{\prime}\right)+\cos \left(\lambda+\nu^{\prime},-\lambda^{\prime}+\mu, \mu^{\prime}+\nu\right)+\cos \left(\lambda+\nu^{\prime}, \lambda^{\prime}+\mu, \nu-\mu^{\prime}\right)+ \\
\left.\cos \left(\lambda+\nu^{\prime}, \lambda^{\prime}+\mu, \mu^{\prime}+\nu\right)\right)
\end{array}
$$

Similarly, the product of two alternating $\operatorname{sines,~} \sin _{(\lambda, \mu, \nu)} \sin _{\left(\lambda^{\prime}, \mu^{\prime}, \nu^{\prime}\right)}$ can be decomposed into the sum of alternating cosines using the following formula:

$$
\begin{aligned}
& \sin _{(\lambda, \mu, \nu)} \sin _{\left(\lambda^{\prime}, \mu^{\prime}, \nu^{\prime}\right)}=\frac{1}{8}\left(\cos _{\left(\lambda-\lambda^{\prime}, \mu-\mu^{\prime}, \nu-\nu^{\prime}\right)}-\cos _{\left(\lambda-\lambda^{\prime}, \mu-\mu^{\prime}, \nu+\nu^{\prime}\right)}-\cos _{\left(\lambda-\lambda^{\prime}, \mu+\mu^{\prime}, \nu-\nu^{\prime}\right)}\right. \\
& +\cos _{\left(\lambda-\lambda^{\prime}, \mu+\mu^{\prime}, \nu+\nu^{\prime}\right)}-\cos _{\left(\lambda+\lambda^{\prime}, \mu-\mu^{\prime}, \nu-\nu^{\prime}\right)}+\cos _{\left(\lambda+\lambda^{\prime}, \mu-\mu^{\prime}, \nu+\nu^{\prime}\right)}+\cos _{\left(\lambda+\lambda^{\prime}, \mu+\mu^{\prime}, \nu-\nu^{\prime}\right)}-\cos _{\left(\lambda+\lambda^{\prime}, \mu+\mu^{\prime}, \nu+\nu^{\prime}\right)} \\
& +\cos _{\left(\lambda-\mu^{\prime}, \mu-\nu^{\prime}, \nu-\lambda^{\prime}\right)}-\cos _{\left(\lambda-\mu^{\prime}, \mu-\nu^{\prime}, \lambda^{\prime}+\nu\right)}-\cos \left(\lambda-\mu^{\prime}, \mu+\nu^{\prime}, \nu-\lambda^{\prime}\right)+\cos _{\left(\lambda-\mu^{\prime}, \mu+\nu^{\prime}, \lambda^{\prime}+\nu\right)}-\cos _{\left(\lambda+\mu^{\prime}, \mu-\nu^{\prime}, \nu-\lambda^{\prime}\right)} \\
& +\cos _{\left(\lambda+\mu^{\prime}, \mu-\nu^{\prime}, \lambda^{\prime}+\nu\right)}+\cos \left(\lambda+\mu^{\prime}, \mu+\nu^{\prime}, \nu-\lambda^{\prime}\right)-\cos \left(\lambda+\mu^{\prime}, \mu+\nu^{\prime}, \lambda^{\prime}+\nu\right)+\cos _{\left(\lambda-\nu^{\prime}, \mu-\lambda^{\prime}, \nu-\mu^{\prime}\right)}-\cos _{\left(\lambda-\nu^{\prime}, \mu-\lambda^{\prime}, \mu^{\prime}+\nu\right)} \\
& -\cos _{\left(\lambda-\nu^{\prime}, \lambda^{\prime}+\mu, \nu-\mu^{\prime}\right)}+\cos _{\left(\lambda-\nu^{\prime}, \lambda^{\prime}+\mu, \mu^{\prime}+\nu\right)}-\cos _{\left(\lambda+\nu^{\prime}, \mu-\lambda^{\prime}, \nu-\mu^{\prime}\right)}+\cos _{\left(\lambda+\nu^{\prime}, \mu-\lambda^{\prime}, \mu^{\prime}+\nu\right)}+\cos _{\left(\lambda+\nu^{\prime}, \lambda^{\prime}+\mu, \nu-\mu^{\prime}\right)} \\
& \left.-\cos _{\left(\lambda+\nu^{\prime}, \lambda^{\prime}+\mu, \mu^{\prime}+\nu\right)}\right) .
\end{aligned}
$$

For the sake of completeness, one can decompose also the product $\sin _{(\lambda, \mu, \nu)} \cos _{\left(\lambda^{\prime}, \mu^{\prime}, \nu^{\prime}\right)}$. This can be however easily obtained from the decomposition (7) by substitution $\cos \rightarrow \sin$ on the right hand side.

\section{DisCRETE COSINE TRANSFORMS}

To each of standard discrete cosine transforms, denoted in the literature often as DCT-1, DCT-2, DCT-3 and DCT-4 [13], there corresponds an alternating three-dimensional discrete cosine transforms. We denote these corresponding transforms as AMDCT-1, AMDCT-2, AMDCT-3, AMDCT-4.

\subsection{AMDCT-1}

For given $N \in \mathbb{N}$ let us define a lattice

$$
F_{N}=\left\{\left(\frac{r}{2 N}, \frac{s}{2 N}, \frac{t}{2 N}\right) \mid 0 \leq r, s, t \leq N, r \geq s \geq t \text { or } s>r>t\right\}
$$




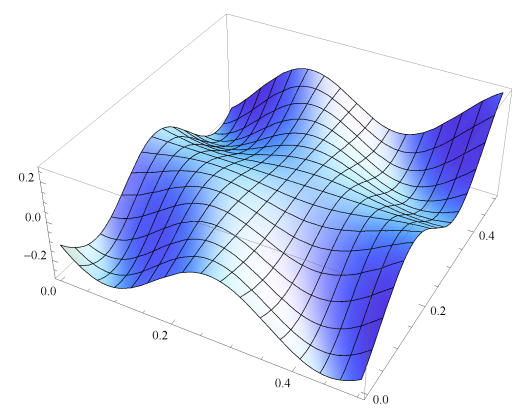

\begin{tabular}{cc}
\hline $\mathrm{N}$ & $\int_{F\left(\tilde{A}_{3}^{\text {aff }}\right)}\left|f-f_{N}\right|^{2}$ \\
\hline 1 & 0.0486711 \\
2 & 0.0391407 \\
3 & 0.0096493 \\
4 & 0.0029516 \\
\hline
\end{tabular}

TABLE 1. Integral error estimates for the approximations in Fig. 4

Figure 3 . The function 10 used in the interpolation example.
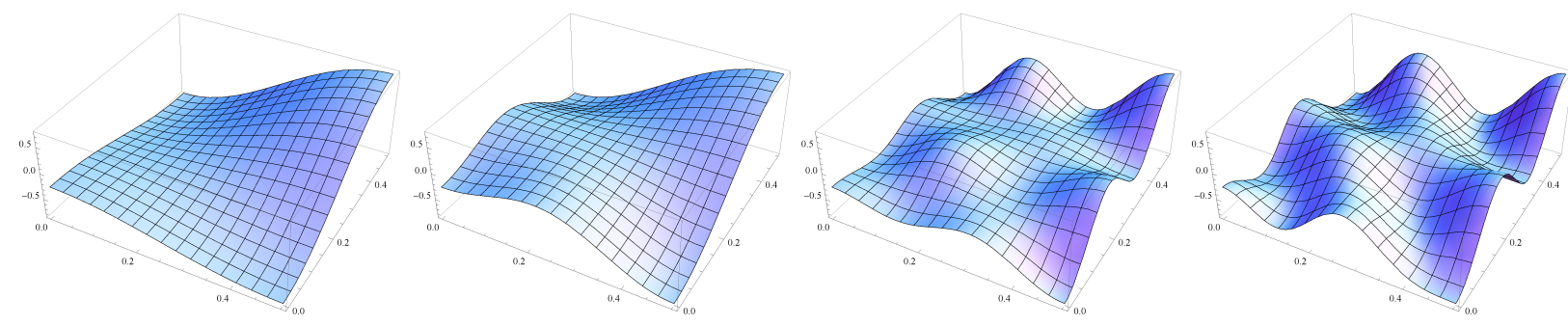

Figure 4. Alternating cosines approximations of 10 for $N=1,2,3,4$.

This lattice is chosen such way that it fulfills the whole space when symmetries (3) and (4) are applied to it. Evidently, we have a partial freedom which parts of boundary to include in the lattice. The lattice (9) contains $\frac{1}{3}\left(N^{3}+3 N^{2}+5 N+3\right)$ points. For example,

$$
F_{2}=\left\{(0,0,0),\left(\frac{1}{4}, 0,0\right),\left(\frac{1}{4}, \frac{1}{4}, 0\right),\left(\frac{1}{4}, \frac{1}{4}, \frac{1}{4}\right),\left(\frac{1}{4}, \frac{1}{2}, 0\right),\left(\frac{1}{2}, 0,0\right),\left(\frac{1}{2}, \frac{1}{4}, 0\right),\left(\frac{1}{2}, \frac{1}{4}, \frac{1}{4}\right),\left(\frac{1}{2}, \frac{1}{2}, 0\right),\left(\frac{1}{2}, \frac{1}{2}, \frac{1}{4}\right),\left(\frac{1}{2}, \frac{1}{2}, \frac{1}{2}\right)\right\} .
$$

Another example, for $N=5$, is shown in Fig. 2

The alternating $\operatorname{cosines} \cos _{(k, l, m)}$ are pairwise orthogonal on this lattice, namely we have

$$
\sum_{\substack{0 \leq r, s, t \leq N, r \geq s \geq t \text { or } s>r>t}} G_{r s t}^{-1} c_{r} c_{s} c_{t} \cos _{(k, l, m)}\left(\frac{r}{2 N}, \frac{s}{2 N}, \frac{t}{2 N}\right) \cos \left(k^{\prime}, l^{\prime}, m^{\prime}\right)\left(\frac{r}{2 N}, \frac{s}{2 N}, \frac{t}{2 N}\right)=N^{3} \tilde{c}_{k} \tilde{c}_{l} \tilde{c}_{m} G_{k l m} \delta_{(k, l, m),\left(k^{\prime}, l^{\prime}, m^{\prime}\right)},
$$

where

$$
c_{\alpha}=\left\{\begin{array}{ll}
\frac{1}{2} & \text { if } \alpha=0 \text { or } \alpha=N, \\
1 & \text { otherwise }
\end{array} \quad \tilde{c}_{\alpha}= \begin{cases}1 & \text { if } \alpha=0 \text { or } \alpha=N, \\
\frac{1}{2} & \text { otherwise }\end{cases}\right.
$$

and $0 \leq k, l, m \leq N, k \geq l \geq m$ or $l>k>m$, and $0 \leq k^{\prime}, l^{\prime}, m^{\prime} \leq N, k^{\prime} \geq l^{\prime} \geq m^{\prime}$ or $l^{\prime}>k^{\prime}>m^{\prime}$.

Let $f$ be a real function defined on $F_{N}$. Then it can be expanded into a sum of alternating cosines,

$$
f(x, y, z)=\sum_{\substack{0 \leq k, l, m \leq N, k \geq l \geq m \text { or } l>k>m}} a_{(k, l, m)} \cos _{(k, l, m)}(x, y, z),
$$

where the coefficients $a_{(k, l, m)}$ are given by the formula

$$
a_{(k, l, m)}=\frac{1}{N^{3} c_{k} c_{l} c_{m} G_{k l m}} \sum_{\substack{0 \leq r, s, t \leq N, r \geq s \geq t \text { or } s>r>t}} \frac{\tilde{c}_{r} \tilde{c}_{s} \tilde{c}_{t}}{G_{r s t}} f\left(\frac{r}{2 N}, \frac{s}{2 N}, \frac{t}{2 N}\right) \cos _{(k, l, m)}\left(\frac{r}{2 N}, \frac{s}{2 N}, \frac{t}{2 N}\right) .
$$

As an interpolation example, we take

$$
f(x, y, z)=\cos 2 \pi x \cos 2 \pi y \cos 2 \pi z \cos 10(x+y+z),
$$

and compute four interpolations $f_{N}$ for $N=1,2,3,4$ using alternating cosines. The graph of the function 100 is shown in Fig. 3 and four approximations in Fig. 4

By visual inspection, approximations $f_{N}$ get closer to $f$ as $N$ increases. This is verified by computing integral error estimates shown in Table 1 


\subsection{AMDCT-2}

Alternating discrete cosine transform of second kind uses a lattice

$$
\tilde{F}_{N}=\left\{\left(\frac{r+1 / 2}{2 N}, \frac{s+1 / 2}{2 N}, \frac{t+1 / 2}{2 N}\right) \mid 0 \leq r, s, t \leq N-1, r \geq s \geq t \text { or } s>r>t\right\} .
$$

This lattice contains $\frac{1}{3} N\left(N^{2}+2\right)$ points. For example,

$$
\tilde{F}_{2}=\left\{\left(\frac{1}{8}, \frac{1}{8}, \frac{1}{8}\right),\left(\frac{3}{8}, \frac{1}{8}, \frac{1}{8}\right),\left(\frac{3}{8}, \frac{3}{8}, \frac{1}{8}\right),\left(\frac{3}{8}, \frac{3}{8}, \frac{3}{8}\right)\right\} .
$$

The alternating $\operatorname{cosines} \cos _{(k, l, m)}$ are pairwise orthogonal on this lattice, namely we have

$$
\begin{aligned}
\sum_{\substack{0 \leq r, s, t \leq N-1, r \geq s \geq t \text { or } s>r>t}} G_{r s t}^{-1} c_{r} c_{s} c_{t} \cos (k, l, m) \\
r N
\end{aligned}
$$

where $0 \leq k, l, m \leq N-1, k \geq l \geq m$ or $l>k>m$, and $0 \leq k^{\prime}, l^{\prime}, m^{\prime} \leq N-1, k^{\prime} \geq l^{\prime} \geq m^{\prime}$ or $l^{\prime}>k^{\prime}>m^{\prime}$.

Let $\tilde{f}$ be a real function defined on $\tilde{F}_{N}$. Then it can be expanded into a sum of alternating cosines,

$$
\tilde{f}(x, y, z)=\sum_{\substack{0 \leq k, l, m \leq N-1, k \geq l \geq m \text { or } l>k>m}} b_{(k, l, m)} \cos (k, l, m)(x, y, z)
$$

where the coefficients $b_{(k, l, m)}$ are given by the formula

$$
b_{(k, l, m)}=\frac{1}{N^{3} c_{k} c_{l} c_{m} G_{k l m}} \sum_{\substack{0 \leq r, s, t \leq N-1, r \geq s \geq t \text { or } \\ s>r>t}} \frac{\tilde{c}_{r} \tilde{c}_{s} \tilde{c}_{t}}{G_{r s t}} \tilde{f}\left(\frac{r+1 / 2}{2 N}, \frac{s+1 / 2}{2 N}, \frac{t+1 / 2}{2 N}\right) \cos (k, l, m)\left(\frac{r+1 / 2}{2 N}, \frac{s+1 / 2}{2 N}, \frac{t+1 / 2}{2 N}\right) .
$$

\subsection{AMDCT-3}

Alternating discrete cosine transform of the third kind uses the lattice

$$
F_{N}^{\prime}=\left\{\left(\frac{r}{2 N}, \frac{s}{2 N}, \frac{t}{2 N}\right) \mid 0 \leq r, s, t \leq N-1, r \geq s \geq t \text { or } s>r>t\right\} .
$$

Instead of alternating cosines $\cos _{(k, l, m)}$, shifted cosines $\cos _{(k+1 / 2, l+1 / 2, m+1 / 2)}$ are used. They have similar properties as normal alternating cosines with integer arguments. For scalar product we obtain

$$
\begin{aligned}
\sum_{\substack{0 \leq r, s, t \leq N-1, r \geq s \geq t \text { or } s>r>t}} G_{r s t}^{-1} c_{r} c_{s} c_{t} \cos _{(k+1 / 2, l+1 / 2, m+1 / 2)}\left(\frac{r}{2 N}, \frac{s}{2 N}, \frac{t}{2 N}\right) \cos _{\left(k^{\prime}+1 / 2, l^{\prime}+1 / 2, m^{\prime}+1 / 2\right)}\left(\frac{r}{2 N}, \frac{s}{2 N}, \frac{t}{2 N}\right) \\
=N^{3} \tilde{c}_{k} \tilde{c}_{l} \tilde{c}_{m} G_{k l m} \delta_{(k, l, m),\left(k^{\prime}, l^{\prime}, m^{\prime}\right)}
\end{aligned}
$$

where $0 \leq k, l, m \leq N-1, k \geq l \geq m$ or $l>k>m$, and $0 \leq k^{\prime}, l^{\prime}, m^{\prime} \leq N-1, k^{\prime} \geq l^{\prime} \geq m^{\prime}$ or $l^{\prime}>k^{\prime}>m^{\prime}$.

Let $g$ be a real function defined on $F_{N}^{\prime}$. Then it can be expanded into a sum of shifted alternating cosines,

$$
g(x, y, z)=\sum_{\substack{0 \leq k, l, m \leq N-1, k \geq l \geq m \text { or } l>k>m}} c_{(k, l, m)} \cos _{(k+1 / 2, l+1 / 2, m+1 / 2)}(x, y, z),
$$

where the coefficients $c_{(k, l, m)}$ are given by the formula

$$
c_{(k, l, m)}=\frac{1}{N^{3} c_{k} c_{l} c_{m} G_{k l m}} \sum_{\substack{0 \leq r, s, t \leq N-1, r \geq s \geq t \text { or } s>r>t}} \frac{\tilde{c}_{r} \tilde{c}_{s} \tilde{c}_{t}}{G_{r s t}} g\left(\frac{r}{2 N}, \frac{s}{2 N}, \frac{t}{2 N}\right) \cos (k+1 / 2, l+1 / 2, m+1 / 2)\left(\frac{r}{2 N}, \frac{s}{2 N}, \frac{t}{2 N}\right) .
$$

\subsection{AMDCT-4}

Alternating discrete cosine transform of the fourth kind uses the lattice $\tilde{F}_{N}$ and shifted cosines as in the third case. We have

$$
\begin{aligned}
& \sum_{\substack{0 \leq r, s, t \leq N-1, r \geq s \geq t \text { or } s>r>t}} G_{r s t}^{-1} c_{r} c_{s} c_{t} \cos _{(k+1 / 2, l+1 / 2, m+1 / 2)}\left(\frac{r+1 / 2}{2 N}, \frac{s+1 / 2}{2 N}, \frac{t+1 / 2}{2 N}\right) \\
& \quad \times \cos _{\left(k^{\prime}+1 / 2, l^{\prime}+1 / 2, m^{\prime}+1 / 2\right)}\left(\frac{r+1 / 2}{2 N}, \frac{s+1 / 2}{2 N}, \frac{t+1 / 2}{2 N}\right)=N^{3} \tilde{c}_{k} \tilde{c}_{l} \tilde{c}_{m} G_{k l m} \delta_{(k, l, m),\left(k^{\prime}, l^{\prime}, m^{\prime}\right)},
\end{aligned}
$$

where $0 \leq k, l, m \leq N-1, k \geq l \geq m$ or $l>k>m$, and $0 \leq k^{\prime}, l^{\prime}, m^{\prime} \leq N-1, k^{\prime} \geq l^{\prime} \geq m^{\prime}$ or $l^{\prime}>k^{\prime}>m^{\prime}$. 
Let $\tilde{f}$ be a real function defined on $\tilde{F}_{N}$. Then it can be expanded into a sum

$$
\tilde{f}(x, y, z)=\sum_{\substack{0 \leq k, l, m \leq N-1, k \geq l \geq m \text { or } l>k>m}} d_{(k, l, m)} \cos (k+1 / 2, l+1 / 2, m+1 / 2)(x, y, z),
$$

where the coefficients $d_{(k, l, m)}$ are given by the formula

$$
d_{(k, l, m)}=\frac{1}{N^{3} c_{k} c_{l} c_{m} G_{k l m}} \sum_{\substack{0 \leq r, s, t \leq N-1, r \geq s \geq t \text { or } \\ s>r>t}} \frac{\tilde{c}_{r} \tilde{c}_{s} \tilde{c}_{t}}{G_{r s t}} \tilde{f}\left(\frac{r+1 / 2}{2 N}, \frac{s+1 / 2}{2 N}, \frac{t+1 / 2}{2 N}\right) \cos (k+1 / 2, l+1 / 2, m+1 / 2)\left(\frac{r+1 / 2}{2 N}, \frac{s+1 / 2}{2 N}, \frac{t+1 / 2}{2 N}\right) .
$$

\section{Discrete SINE TRANSFORMS}

Analogical formulas for discrete sine transforms 1-4 can be derived. To each of standard discrete sine transforms, denoted in the literature often as DST-1, DST-2, DST-3 and DST-4, there corresponds an alternating threedimensional discrete sine transforms. We denote these corresponding transforms as AMDST-1, AMDST-2, AMDST-3, AMDST-4.

\subsection{AMDST-1}

For given $N \in \mathbb{N}$ let us define a lattice

$$
F_{N}^{(S)}=\left\{\left(\frac{r}{2 N}, \frac{s}{2 N}, \frac{t}{2 N}\right) \mid 1 \leq r, s, t \leq N-1, r \geq s \geq t \text { or } s>r>t\right\} .
$$

This lattice contains $\frac{1}{3}\left(N^{3}-3 N^{2}+5 N-3\right)$ points. For example,

$$
F_{3}^{(S)}=\left\{\left(\frac{1}{6}, \frac{1}{6}, \frac{1}{6}\right),\left(\frac{1}{3}, \frac{1}{6}, \frac{1}{6}\right),\left(\frac{1}{3}, \frac{1}{3}, \frac{1}{6}\right),\left(\frac{1}{3}, \frac{1}{3}, \frac{1}{3}\right)\right\} .
$$

The alternating $\operatorname{sines} \sin _{(k, l, m)}$ are pairwise orthogonal on this lattice, namely we have

$$
\sum_{\substack{1 \leq r, s, t \leq N-1, r \geq s \geq t \text { or } s>r>t}} G_{r s t}^{-1} \sin _{(k, l, m)}\left(\frac{r}{2 N}, \frac{s}{2 N}, \frac{t}{2 N}\right) \sin _{\left(k^{\prime}, l^{\prime}, m^{\prime}\right)}\left(\frac{r}{2 N}, \frac{s}{2 N}, \frac{t}{2 N}\right)=\left(\frac{N}{2}\right)^{3} G_{k l m} \delta_{(k, l, m),\left(k^{\prime}, l^{\prime}, m^{\prime}\right)},
$$

where $1 \leq k, l, m \leq N-1, k \geq l \geq m$ or $l>k>m$, and $1 \leq k^{\prime}, l^{\prime}, m^{\prime} \leq N-1, k^{\prime} \geq l^{\prime} \geq m^{\prime}$ or $l^{\prime}>k^{\prime}>m^{\prime}$.

Let $f^{(S)}$ be a real function defined on $F_{N}^{(S)}$. Then it can be expanded into a sum of alternating sines,

$$
f^{(S)}(x, y, z)=\sum_{\substack{1 \leq k, l, m \leq N-1, k \geq l \geq m \text { or } l>k>m}} a_{(k, l, m)} \sin _{(k, l, m)}(x, y, z)
$$

where the coefficients $a_{(k, l, m)}^{(S)}$ are given by the formula

$$
a_{(k, l, m)}^{(S)}=\frac{8}{N^{3} G_{k l m}} \sum_{\substack{1 \leq r, s, t \leq N-1, r \geq s \geq t \text { or } s>r>t}} \frac{1}{G_{r s t}} f^{(S)}\left(\frac{r}{2 N}, \frac{s}{2 N}, \frac{t}{2 N}\right) \sin _{(k, l, m)}\left(\frac{r}{2 N}, \frac{s}{2 N}, \frac{t}{2 N}\right) .
$$

As an interpolation example, we take

$$
f^{(S)}(x, y, z)=\sin 2 \pi x \sin 2 \pi y \sin 2 \pi z \sin 10(x+y+z)
$$

and compute four interpolations $f_{N}^{(S)}$ for $N=1,2,3,4$ using alternating sines. Picture of 12 is shown in Fig. 5 and four approximations in Fig. 6.

By visual inspection, approximations $f_{N}^{(S)}$ get closer to 12 as $N$ increases. This is verified by computing integral error estimates shown in Table 2

\subsection{AMDST-2}

Alternating discrete sine transform of second kind uses the same lattice as AMDCT-2, that is

$$
\tilde{F}_{N}^{(S)}=\tilde{F}_{N}
$$

where $\tilde{F}_{N}$ is given by 11 . 


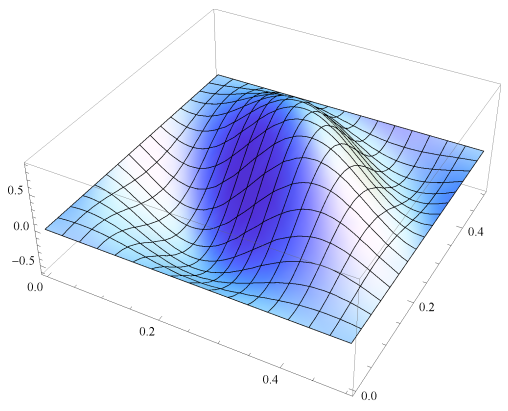

\begin{tabular}{cc}
\hline $\mathrm{N}$ & $\int_{F\left(\tilde{A}_{3}^{\text {aff }}\right)}\left|f^{(S)}-f_{N}^{(S)}\right|^{2}$ \\
\hline 1 & 0.0482575 \\
2 & 0.0282054 \\
3 & 0.0078227 \\
4 & 0.0006646 \\
\hline
\end{tabular}

TABLE 2. Integral error estimates for the approximations in Fig. 6

FiguRE 5. The function 12 used in interpolation example.
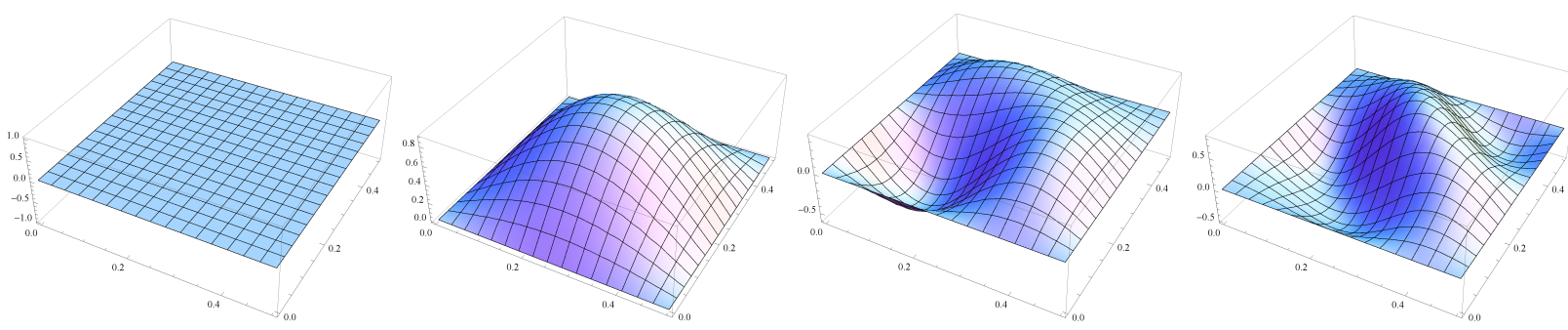

Figure 6. The alternating sines approximations of 12 for $N=1,2,3,4$.

The alternating $\operatorname{sines} \sin _{(k, l, m)}$ are pairwise orthogonal on this lattice, namely we have

$$
\begin{array}{r}
\sum_{\substack{0 \leq r, s, t \leq N-1, r \geq s \geq t \text { or } s>r>t}} G_{r s t}^{-1} \sin _{(k, l, m)}\left(\frac{r+1 / 2}{2 N}, \frac{s+1 / 2}{2 N}, \frac{t+1 / 2}{2 N}\right) \sin _{\left(k^{\prime}, l^{\prime}, m^{\prime}\right)}\left(\frac{r+1 / 2}{2 N}, \frac{s+1 / 2}{2 N}, \frac{t+1 / 2}{2 N}\right) \\
=\left(\frac{N}{2}\right)^{3} c_{k} c_{l} c_{m} G_{k l m} \delta_{(k, l, m),\left(k^{\prime}, l^{\prime}, m^{\prime}\right)},
\end{array}
$$

where $c_{p}=\frac{1}{2}$ for $p=N$ and $c_{p}=1$ otherwise and $1 \leq k, l, m \leq N, k \geq l \geq m$ or $l>k>m$, and $1 \leq k^{\prime}, l^{\prime}, m^{\prime} \leq N, k^{\prime} \geq l^{\prime} \geq m^{\prime}$ or $l^{\prime}>k^{\prime}>m^{\prime}$.

Let $\tilde{f}^{(S)}$ be a real function defined on $\tilde{F}_{N}^{(S)}$. Then it can be expanded into a sum of alternating sines,

$$
\tilde{f}^{(S)}(x, y, z)=\sum_{\substack{1 \leq k, l, m \leq N, k \geq l \geq m \text { or } l>k>m}} b_{(k, l, m)}^{(S)} \sin _{(k, l, m)}(x, y, z),
$$

where the coefficients $b_{(k, l, m)}$ are given by the formula

$$
b_{(k, l, m)}^{(S)}=\frac{8}{N^{3} c_{k} c_{l} c_{m} G_{k l m}} \sum_{\substack{0 \leq r, s, t \leq N-1, r \geq s \geq t \text { or } s>r>t}} \frac{1}{G_{r s t}} \tilde{f}^{(S)}\left(\frac{r+1 / 2}{2 N}, \frac{s+1 / 2}{2 N}, \frac{t+1 / 2}{2 N}\right) \sin _{(k, l, m)}\left(\frac{r+1 / 2}{2 N}, \frac{s+1 / 2}{2 N}, \frac{t+1 / 2}{2 N}\right) .
$$

\subsection{AMDST-3}

Alternating discrete sine transform of the third kind uses the lattice

$$
F_{N}^{\prime(S)}=\left\{\left(\frac{r}{2 N}, \frac{s}{2 N}, \frac{t}{2 N}\right) \mid 1 \leq r, s, t \leq N, r \geq s \geq t \text { or } s>r>t\right\} .
$$

Instead of alternating $\operatorname{sines} \sin _{(k, l, m)}$, shifted $\operatorname{sines} \sin _{(k+1 / 2, l+1 / 2, m+1 / 2)}$ are used. They have similar properties as normal alternating sines with integer arguments. For scalar product we obtain

$$
\begin{aligned}
& \sum_{\substack{1 \leq r, s, t \leq N, r \geq s \geq t \text { or } s>r>t}} G_{r s t}^{-1} c_{r} c_{s} c_{t} \sin _{(k+1 / 2, l+1 / 2, m+1 / 2)}\left(\frac{r}{2 N}, \frac{s}{2 N}, \frac{t}{2 N}\right) \sin _{\left(k^{\prime}+1 / 2, l^{\prime}+1 / 2, m^{\prime}+1 / 2\right)}\left(\frac{r}{2 N}, \frac{s}{2 N}, \frac{t}{2 N}\right) \\
& =\left(\frac{N}{2}\right)^{3} G_{k l m} \delta_{(k, l, m),\left(k^{\prime}, l^{\prime}, m^{\prime}\right)}
\end{aligned}
$$

where $c_{p}=\frac{1}{2}$ for $p=N$ and $c_{p}=1$ otherwise and $0 \leq k, l, m \leq N-1, k \geq l \geq m$ or $l>k>m$, and $0 \leq k^{\prime}, l^{\prime}, m^{\prime} \leq N-1, k^{\prime} \geq l^{\prime} \geq m^{\prime}$ or $l^{\prime}>k^{\prime}>m^{\prime}$. 
Let $g^{(S)}$ be a real function defined on $F_{N}^{\prime}{ }^{(S)}$. Then it can be expanded into a sum of shifted alternating sines,

$$
g^{(S)}(x, y, z)=\sum_{\substack{0 \leq k, l, m \leq N-1, k \geq l \geq m \text { or } l>k>m}} c_{(k, l, m)}^{(S)} \sin _{(k+1 / 2, l+1 / 2, m+1 / 2)}(x, y, z)
$$

where the coefficients $c_{(k, l, m)}^{(S)}$ are given by the formula

$$
c_{(k, l, m)}^{(S)}=\frac{8}{N^{3} G_{k l m}} \sum_{\substack{1 \leq r, s, t \leq N, r \geq s \geq t \text { or } s>r>t}} \frac{c_{r} c_{s} c_{t}}{G_{r s t}} g^{(S)}\left(\frac{r}{2 N}, \frac{s}{2 N}, \frac{t}{2 N}\right) \sin _{(k+1 / 2, l+1 / 2, m+1 / 2)}\left(\frac{r}{2 N}, \frac{s}{2 N}, \frac{t}{2 N}\right) .
$$

\subsection{AMDST-4}

Alternating discrete sine transform of the fourth kind uses the lattice $\tilde{F}_{N}^{(S)}$ given by 13 and shifted sines as in the third case. We have

$$
\begin{array}{r}
\sum_{\substack{0 \leq r, s, t \leq N-1, r \geq s \geq t \text { or } s>r>t}} G_{r s t}^{-1} \sin _{(k+1 / 2, l+1 / 2, m+1 / 2)}\left(\frac{r+1 / 2}{2 N}, \frac{s+1 / 2}{2 N}, \frac{t+1 / 2}{2 N}\right) \sin _{\left(k^{\prime}+1 / 2, l^{\prime}+1 / 2, m^{\prime}+1 / 2\right)}\left(\frac{r+1 / 2}{2 N}, \frac{s+1 / 2}{2 N}, \frac{t+1 / 2}{2 N}\right) \\
=\left(\frac{N}{2}\right)^{3} G_{k l m} \delta_{(k, l, m),\left(k^{\prime}, l^{\prime}, m^{\prime}\right)},
\end{array}
$$

where $0 \leq k, l, m \leq N-1, k \geq l \geq m$ or $l>k>m$, and $0 \leq k^{\prime}, l^{\prime}, m^{\prime} \leq N-1, k^{\prime} \geq l^{\prime} \geq m^{\prime}$ or $l^{\prime}>k^{\prime}>m^{\prime}$.

Let $\tilde{f}^{(S)}$ be a real function defined on $\tilde{F}_{N}^{(S)}$. Then it can be expanded into a sum

$$
\tilde{f}^{(S)}(x, y, z)=\sum_{\substack{0 \leq k, l, m \leq N-1, k \geq l \geq m \text { or } l>k>m}} d_{(k, l, m)}^{(S)} \sin _{(k+1 / 2, l+1 / 2, m+1 / 2)}(x, y, z)
$$

where the coefficients $d_{(k, l, m)}^{(S)}$ are given by the formula

$$
d_{(k, l, m)}^{(S)}=\frac{8}{N^{3} G_{k l m}} \sum_{\substack{0 \leq r, s, t \leq N-1, r \geq s \geq t \text { or } s>r>t}} \frac{1}{G_{r s t}} \tilde{f}\left(\frac{r+1 / 2}{2 N}, \frac{s+1 / 2}{2 N}, \frac{t+1 / 2}{2 N}\right) \sin (k+1 / 2, l+1 / 2, m+1 / 2)\left(\frac{r+1 / 2}{2 N}, \frac{s+1 / 2}{2 N}, \frac{t+1 / 2}{2 N}\right) .
$$

\section{Conclusion}

We have presented a detailed study of alternating three dimensional trigonometric functions and their properties including product decomposition, continuous and discrete orthogonality and interpolation problem. Practical computational aspects of the formalism presented in this paper need further investigation, namely a fast Fourier transform analog and comparing to the usual discrete Fourier transform in three dimensions.

\section{ACKNOWLEDGEMENTS}

We gratefully acknowledge the support of this work by the Doppler Institute of the Czech Technical University in Prague. $\mathrm{AB}$ is grateful for the hospitality extended to her at Department of mathematics FNSPE CTU. SP acknowledges the support of SGS15/215/OHK4/3T/14, project of the Czech Technical University in Prague.

\section{REFERENCES}

[1] A. Klimyk, J. Patera. (Anti)symmetric multivariate exponential functions and corresponding Fourier transforms. $J$ Phys A 40(34):10473-10489, 2007. DOI:10.1088/1751-8113/40/34/006.

[2] A. Bezubik, J. Hrivnák, J. Patera, S. Pošta. Three variable symmetric and antisymmetric exponential functions and orthogonal polynomials. Math Slovaca To appear.

[3] A. Klimyk, J. Patera. Alternating group and multivariate exponential functions. In Groups and symmetries, vol. 47 of CRM Proc. Lecture Notes, pp. 233-246. Amer. Math. Soc., Providence, RI, 2009.

[4] J. Hrivnák, J. Patera, S. Pošta. Three-variable exponential functions of the alternating group. J Phys $A$ 45(4):045201, 10, 2012. DOI:10.1088/1751-8113/45/4/045201

[5] A. Klimyk, J. Patera. (Anti)symmetric multivariate trigonometric functions and corresponding Fourier transforms. $J$ Math Phys 48(9):093504, 24, 2007. DOI:10.1063/1.2779768.

[6] A. Klimyk, J. Patera. Alternating multivariate trigonometric functions and corresponding Fourier transforms. $J$ Phys A 41(14):145205, 16, 2008. DOI:10.1088/1751-8113/41/14/145205.

[7] J. Hrivnák, J. Patera. Two-dimensional symmetric and antisymmetric generalizations of exponential and cosine functions. J Math Phys 51(2):023515, 24, 2010. DOI:10.1063/1.3282850. 
[8] J. Hrivnák, L. Motlochová, J. Patera. Two-dimensional symmetric and antisymmetric generalizations of sine functions. J Math Phys 51(7):073509, 13, 2010. DOI:10.1063/1.3430567.

[9] A. Klimyk, J. Patera. Orbit functions. SIGMA Symmetry Integrability Geom Methods Appl 2:Paper 006, 60, 2006. DOI:10.3842/SIGMA.2006.006.

[10] A. Klimyk, J. Patera. Antisymmetric orbit functions. SIGMA Symmetry Integrability Geom Methods Appl 3:Paper 023, 83, 2007. DOI:10.3842/SIGMA.2007.023.

[11] A. Klimyk, J. Patera. E-orbit functions. SIGMA Symmetry Integrability Geom Methods Appl 4:Paper 002, 57, 2008. DOI:10.3842/SIGMA.2008.002.

[12] H. Minc. Permanents, vol. 9999 of Encyclopedia of Mathematics and its Applications. Addison-Wesley Publishing Co., Reading, Mass., 1978. With a foreword by Marvin Marcus, Encyclopedia of Mathematics and its Applications, Vol. 6.

[13] K. R. Rao, P. Yip. Discrete cosine transform. Academic Press Inc., Boston, MA, 1990. Algorithms, advantages, applications. 